Communications in Physics, Vol. 26, No. 2 (2016), pp. 181-192

DOI: $10.15625 / 0868-3166 / 26 / 2 / 7866$

\title{
KLEIN-GORDON-FOCK EQUATION FROM EINSTEIN GENERAL RELATIVITY
}

\author{
VO VAN THUAN ${ }^{\dagger}$ \\ Vietnam Atomic Energy Institute (VINATOM), \\ 59 Ly Thuong Kiet street, Hoan Kiem district, Hanoi, Vietnam \\ ${ }^{\dagger}$ E-mail: vvthuan@vinatom.gov.vn \\ Received 10 March 2016 \\ Accepted for publication 10 September 2016
}

\begin{abstract}
A time-space symmetry based cylindrical model of geometrical dynamics was proposed. Accordingly, the solution of Einstein gravitational equation in vacuum has a duality: an exponential solution and a wave-like one. The former leads to a "microscopic" cosmological model with Hubble expansion. Due to interaction of a Higgs-like cosmological potential, the original time-space symmetry is spontaneously broken, inducing a strong time-like curvature and a weak space-like deviation curve. In the result, the wave-like solution leads to Klein-Gordon-Fock equation which would serve an explicit approach to the problem of consistency between quantum mechanics and general relativity.
\end{abstract}

Keywords: time-space symmetry, general relativity, microscopic cosmological model, wave-like solution, Klein-Gordon-Fock equation, physical reality, wave-particle duality.

Classification numbers: 03.65.-w; 04.90.+e; 04.50.Cd.

\section{INTRODUCTION}

Searching for the consistency between quantum mechanics and general relativity is the most important and long-standing issue of physics. Kaluza and Klein [1,2] were pioneers to propose a space-like extradimension (ED) which is compacted as a micro circle in relation to general relativity. For the semi-classical approach to quantum mechanics introduced by de Broglie and Bohm [3,4] the hidden parameters are somehow reminiscent of EDs. However, the evidence for violation of Bell inequalities [5,6] abandoned the models with local hidden parameters, leaving the door still open to non-local hidden variables. High dimensional superstring models have been developed following Kaluza-Klein geometrical dynamics, of which most applied space-like EDs, while few others considered time-like ones. There are two main approaches applying time-like EDs: membrane models in the Anti-de-Sitter geometry (AdS), such as [7,8] and induced matter 
models [9, 10]. In particular, Maldacena [7] found a duality between AdS and conformal fields as AdS/CFT formalism. Randall and Sundrum [8] applied an infinite AdS 5D-model for a hierarchy solution. For the induced matter approach, Wesson [9] has proposed a space-time-matter model (STM) describing proper mass as a time-like ED. The STM co-workers have proposed a null geodesics model of extended general relativity in 5D manifolds [10], which in a link with 4D space-time physics of elementary particles would lead to a qualitative interpretation of important issues of quantum mechanics, such as wave-particle duality, a wave-like origin of mass and Heisenberg indeterminism [11]. However, they considered that cylindrical curvature is a too strong constraint. A geometrical dynamic model for elementary particles was proposed by Koch [12] with a time-like ED which offered a method for derivation of Klein-Gordon equation. Following the induced-matter approach our preliminary study [13] was based on the time-space symmetry in which the Klein-Fock reduction formalism was used and the two time-like extra-dimensions were made explicit in terms of the quantum wave function $\psi$ and the proper time variable $t_{0}$. For a next step [14], a duality was found between the quantum wave equations in 4D space-time and a relativistic geodesic description of the curved extradimensional time-space and, as a result, Heisenberg indeterminism is shown to originate from the space-time curvatures. Concerning the experimental verification, applying 3D-extended time-like curvatures can solve quantitatively the problem of mass hierarchy of charge lepton generations [15] which opens a new possibility for extending to a more general solution of the heavy lepton-neutrino hierarchy. It would be an advantage of our cylindrical dynamical model at variance with more general approaches of other induced matter models. The goal of the present work is to look for a direct approach to the consistency between quantum mechanics and general relativity which would be implemented in particle physics. We are going to show that the extended general relativity equation leads to the desirable geodesic equation, which is mathematically identical to the basic Klein-Gordon-Fock quantum equation of free massive elementary particles. This duality of Einstein gravitational equation is able to shed light on physical reality of quantum substances.

\section{TIME-SPACE SYMMETRICAL GEOMETRY}

Considering the flat $\{3 T, 3 X\} \equiv\left\{t_{1}, t_{2}, t_{3} \mid x_{1}, x_{2}, x_{3}\right\}$ symmetrical time-space:

$$
d S^{2}=d t_{k}^{2}-d x_{l}^{2}
$$

where $k, l=1 \div 3$ are summation indices. Here we use mostly natural units throughout except when it needs involving quantum dimension. The physics is investigated on the 6D-"lightcone" of time-space (1):

$$
d t_{k}^{2}=d x_{l}^{2}
$$

In particular, considering in the lightcone manifold (2) harmonic correlations $\psi_{0}\left[d t_{k}, d x_{l}\right]$ between a time-like and a space-like differentials-displacements as:

$$
\frac{\partial^{2} \psi_{0}}{\partial t_{k}^{2}}=\frac{\partial^{2} \psi_{0}}{\partial x_{l}^{2}}
$$

We assume that those homogenous and isotropic plane waves serve a primitive source of quantum fluctuations in space-time. All chaos of displacements $d t_{k}$ and $d x_{l}$ can form square-averaged timelike and space-like potentials $V_{T}$ and $V_{X}$, respectively.

For description of homogeneous and isotropic fluctuations a spherical geometry is available. We 
suggest that the global potentials, originally accelerating $\psi_{0}\left(t_{k}, x_{l}\right)$, turn plane waves into geodesic deviations with time-space symmetrical bi-spherical curvatures which are equivalent to isotropic spinning in symmetrical orthonormal subspaces of 3D-time and 3D-space. For elementary particles, except 3D-spatial spin $\vec{s}$, a pseudo-isospin $\vec{\tau}$ is introduced in 3D-time sphere. Therefore, the bi-spherical geometry with variables $\{\psi, \theta, \varphi\}$ reads

$$
d \Sigma^{2}=d s^{2}-d \sigma^{2}=d t^{2}-d \lambda^{2}
$$

where

$$
d t^{2}=d \psi\left(t_{k}\right)^{2}+\psi\left(t_{k}\right)^{2}\left[d \theta\left(t_{k}\right)^{2}+\sin ^{2} \theta\left(t_{k}\right) d \varphi\left(t_{k}\right)^{2}\right]
$$

and

$$
d \lambda^{2}=d \psi\left(x_{l}\right)^{2}+\psi\left(x_{l}\right)^{2}\left[d \theta\left(x_{l}\right)^{2}+\sin ^{2} \theta\left(x_{l}\right) d \varphi\left(x_{l}\right)^{2}\right] .
$$

The original spherical coordinates $\{\psi, \theta, \varphi\}$ of Geometry (4) being embedded in manifold (2) are getting then functionally depending on linear coordinates $\left\{t_{k}, x_{l}\right\}$.

For fluctuations including curved rotation and linear translation a $\{3 T, 3 X\}$-symmetrical bi-cylindrical geometry is applicable as a simplest model. In particular, for an individual fermion elementary particle, e.g. a free lepton with its (pseudo-)spins $\vec{\tau}$ and/or spin $\vec{s}$ being fixed on an arbitrary axis relative to the longitudinal translational axes of $\left\{t_{k}\right\}$ and $\left\{x_{l}\right\}$, respectively, then the $3 \mathrm{D}$-spherical rotation is reduced to the 2D-cylindrical spinning. However, except their longitudinal projections $\tau_{k}$ or $s_{l}= \pm 1 / 2$ following strictly the cylindrical condition with: $d \theta^{2}=0$ and $\sin ^{2} \theta=$ 1 , there are existing also other longitudinal fluctuations due to perpendicular projections of spin and pseudo-spin. This dynamical model leads to a general time-space symmetrical bi-cylindrical geometry

$$
d \Sigma^{2}=\left(d s_{0}^{2}+d s_{e v}^{2}+d s_{\text {long }}^{2}\right)-\left(d \sigma_{e v}^{2}+d \sigma_{\text {long }}^{2}+d \sigma_{L}^{2}\right)=d t^{2}-d \lambda^{2},
$$

where

$$
d t^{2}=d \psi\left(t_{0}, t_{k}\right)^{2}+\psi\left(t_{0}, t_{k}\right)^{2} d \varphi\left(t_{0}, t_{k}\right)^{2}+d t_{k}^{2}
$$

and

$$
d \lambda^{2}=d \psi\left(x_{n}, x_{l}\right)^{2}+\psi\left(x_{n}, x_{l}\right)^{2} d \varphi\left(x_{n}, x_{l}\right)^{2}+d x_{l}^{2} ;
$$

and a contribution from longitudinal fluctuations is expressed through the additional intervals $d s_{\text {long }}$ and $d \sigma_{\text {long }}$ which should be somehow neutralized for conservation of Lorentz invariance. Here the symmetrical curved time-space $\{3 T, 3 X\} \equiv\left\{\psi\left(t_{0}, t_{k}\right), \varphi\left(t_{0}, t_{k}\right), t_{k} \mid \psi\left(x_{n}, x_{l}\right), \varphi\left(x_{n}, x_{l}\right), x_{l}\right\}$. In a semi-phenomenological consideration, the T-odd term $d s_{0}$ is equivalent to a conventional interval of special relativity in $4 \mathrm{D}$-Minkowski space-time, while the P-odd term $d \sigma_{L}$ is a $\mathrm{P}$ nonconserving contribution (PNC) of the weak interaction making a global space-like curvature in term of the left-handed helicity of fermion elementary particles. The P-even term $d \sigma_{e v}$ describes 3D-space kinetics then $x_{3}$ is selected as a cylindrical axis, implying that $x_{3} \in\left\{x_{l}\right\}$. The time-like even-term $d s_{e v} \equiv d \sigma_{C P V}$ is introduced to describe CP-violation effects in 3D-time. Being embedded in Manifold (2) the cylindrical variables $\psi$ and $\varphi$ are getting functions of linear coordinates $\left\{t_{k}, x_{l}\right\}$ and two 3D-local affine parameters $t_{0}$ and $x_{n}$ which are introduced in according to the longitudinal projection of (pseudo-)spins $\vec{\tau}$ and $\vec{s}$, respectively, namely:

$$
\psi=\psi\left(t_{0}, t_{k}, x_{n}, x_{l}\right) ; \quad \varphi=\Omega_{0} t_{0}+\Omega_{k} t_{k}-k_{n} x_{n}-k_{l} x_{l}=\Omega_{i} t_{i}-k_{j} x_{j},
$$


where $\{i, j\}$ are summation indices of curved coordinates. When (pseudo-)spins $\vec{\tau}$ and/or $\vec{s}$ are fixed along the longitudinal translational axes, the bi-cylindrical geometry is getting simpler without longitudinal components of fluctuation. For an explicit description, $\left\{t_{3}, x_{3}\right\}$ are accepted in Geometry (5) as longitudinal central axes of the bi-cylinder, the curved coordinates of 3D-space are $\left\{x_{j}\right\} \in\left\{x_{1}, x_{2}, z\right\}$ with $k^{2} d z^{2}=k_{n}^{2} d x_{n}^{2}+k_{3}^{2} d x_{3}^{2}$. Similarly, for 3D-time there are $\left\{t_{i}\right\} \in\left\{t_{1}, t_{2}, t\right\}$ where the longitudinal axis $t_{3}$ can combine with the rotational affine parameter $t_{0}$ to form the real physical time $t$ by an orthogonal relationship: $\Omega^{2} d t^{2}=\Omega_{0}^{2} d t_{0}^{2}+\Omega_{3}^{2} d t_{3}^{2}$.

Practically, in the following scenario we make an assumption that due to interaction of a Higgslike potential the time-space symmetry is spontaneously broken, leading to formation of energymomentum, which turns the spherical curvature into an almost exact intrinsic cylindrical curvature, when an "internal observer" cannot see any external 3D-subspaces except the involving him curved evolutional axes $\{t, z\}$. In the result, Geometry (5) turns to a more realistic asymmetrical bicylindrical geometry

$$
d \Sigma^{2}=\left(d s_{0}^{2}+d s_{e v}^{2}\right)-\left(d \sigma_{e v}^{2}+d \sigma_{L}^{2}\right)=d t^{2}-d z^{2}
$$

where

and

$$
d t^{2}=d \psi\left(t_{0}\right)^{2}+\psi\left(t_{0}\right)^{2} d \varphi\left(t_{0}\right)^{2}+d t_{3}^{2}
$$

$$
d z^{2}=d \psi\left(x_{n}\right)^{2}+\psi\left(x_{n}\right)^{2} d \varphi\left(x_{n}\right)^{2}+d x_{3}^{2} .
$$

The asymmetrical curved time-space $\{3 T, 3 X\} \equiv\left\{\psi\left(t_{0}\right), \varphi\left(t_{0}\right), t_{3} \mid \psi\left(x_{n}\right), \varphi\left(x_{n}\right), x_{3}\right\}$. The timelike and space-like intervals in Geometry (7) separate into even and odd constituents, which means the corresponding cylindrical accelerations can flip for and back (as an even-term) or cannot flip (as an odd-term) in relation to the cylindrical axis. Odd-terms are determined by internal curvatures, while even-terms relate to external curvatures of cylinders embedded in corresponding flat 3D-subspaces. Naturally, an internal observer involved in a spiral rotation, being not able to distinguish this cylindrical curvature, considers its space-time flat. In principle, a free electron reserves its angular momentum (spin) correlating with fixed $d \sigma_{e v}$, but in an external laboratory frame because the proper angular momentum is no more observable unless using an appropriate on-line polarization analysis, then $d \sigma_{e v}$ is getting hidden by a geodesic compensation. In the result, all three linear spatial axes $\left\{x_{l}\right\}$ reveals in transformation from the cylindrical frame to 3Dspace laboratory frame. In 4D-Minkowski space-time the P-odd term is too small as $d \sigma_{L} \ll d \sigma_{e v}$, that makes the weakly curved $\left\{x_{j}\right\} \approx\left\{x_{l}\right\}$ which open as it is observable in a flat 3D-space. The time-like even-term $d s_{e v} \equiv d \sigma_{C P V}$ is introduced to describe CP-violation effects in 3D-time. Therefore, an asymmetry of Geometry (7) means that in 4D-Minkowski subluminal space-time $d \sigma_{C P V} \ll d \sigma_{L} \ll d \sigma_{e v} \ll d s_{0}$, that $d \sigma_{C P V}$ and $d \sigma_{L}$ may be ignored. It makes Geometry (7) turned to $4 \mathrm{D}$ space-time geometry as:

$$
d \Sigma^{2} \approx d s_{0}^{2}-d \sigma_{e v}^{2}=d t^{2}-d \psi\left(x_{n}\right)^{2}+\psi\left(x_{n}\right)^{2} d \varphi\left(x_{n}\right)^{2}+d x_{3}^{2},
$$

where despite $d t^{2}=d \psi\left(t_{0}\right)^{2}+\psi\left(t_{0}\right)^{2} d \varphi\left(t_{0}\right)^{2}+d t_{3}^{2}$, this curved axis should be observed as linear time for $4 \mathrm{D}$ space-time observers. In the result, the 3D-time internal curvature is getting absolute and the subluminal physics is involved in evolution along the physical time $t$, in the meantime, $d \sigma_{e v}$ turns to a pseudo-cylindrical interval in an almost linear 3D-space $\left\{x_{j}\right\}$ with a weakly curved residue along axis $z$. The curvatures are described by $\operatorname{EDs} \psi$ and $\varphi$ in (6). When there is not a 
polarizer for observation in 3D-space (when the 4D-observer gets off from an internal observation back to 3D-space), the spatial spinning is compensated by the space-like pseudo-cylindrical curvature that the 3D-space in (8) is getting absolutely flat, such as:

$$
d \Sigma^{2} \approx d s_{0}^{2}=d t^{2}-\left[d x_{1}^{2}+d x_{2}\right]-d x_{3}^{2} \equiv d t^{2}-d x_{l}^{2},
$$

where $\left[d x_{1}^{2}+d x_{2}\right]$ is added to explicit that $x_{3} \in\left\{x_{l}\right\}$ in the original flat 3D-space. The 4Dsubluminal geometries (8) and (9) fit the charged lepton sector [15].

For 4D-superluminal time-space, it is assumed that $d \sigma_{e v} \ll d s_{0} \ll d \sigma_{C P V} \ll d \sigma_{L}$ then 3D-time is almost flat, while $d \sigma_{e v}$ and $d s_{0}$ may be ignored. In the result, the 3D-spatial curvature is getting absolute and superluminal substances are to move along the physical 3D-curved $z$; in the meantime, for subluminal observer $d \sigma_{C P V} \ll d \sigma_{L}$ then $d \sigma_{C P V}$ turns to a pseudo-cylindrical interval in an almost flat 3D-time, as from Geometry (7) it turns to 4D time-space geometry as:

$$
d \Sigma^{2} \approx d s_{e v}^{2}-d \sigma_{L}^{2}=d \psi\left(t_{0}\right)^{2}+\psi\left(t_{0}\right)^{2} d \varphi\left(t_{0}\right)^{2}+d t_{3}^{2}-d z^{2},
$$

where similarly, despite $d z^{2}=d \psi\left(x_{n}\right)^{2}+\psi\left(x_{n}\right)^{2} d \varphi\left(x_{n}\right)^{2}+d x_{3}^{2}$, the curved axis $d z$ should be observed as linear space-line for 4D space-time observer, involved in the global weak interaction through left-handed electrons of their bodies. It is usually difficult to observe a $\mathrm{CP}$ violation effect, then the even-term $d s_{e v} \equiv d \sigma_{C P V}$ in (10) is compensated by the time-like pseudo-cylindrical curvature and 3D-time is getting absolutely flat for superluminal substances, that Geometry (10) is simplified:

$$
d \Sigma^{2} \approx-d \sigma_{L}^{2}=\left[d t_{1}^{2}+d t_{2}^{2}\right]+d t_{3}^{2}-d z^{2} \equiv d t_{k}^{2}-d z^{2},
$$

where again $\left[d t_{1}^{2}+d t_{2}^{2}\right]$ is introduced to re-establish the original flat $3 \mathrm{D}$-time. It is to mention that as $4 \mathrm{D}$ subluminal observers we can see only those superluminal leptons which evolve along $t_{3}$ axis partly coinciding with us. In appearance, the superluminal leptons would be seen as luxons moving with speed of light due to a very weak P-odd curvature. The strong asymmetry between space-like and time-like curvatures, as well as a possibility of exchanging the roles of space $\Leftrightarrow$ time (or equivalently, momentum $\Leftrightarrow$ energy) in geometries (10) and (11) in comparison with 4Dsubluminal geometries (8) and (9) would be able to fit neutrino sector, in particular, for solving the problem of large hierarchy between neutrinos and heavy leptons.

\section{A DUALITY OF EINSTEIN GRAVITATIONAL EQUATION}

Suggesting that in both orthonormal subspaces of 3D-time and 3D-space cylindrical curvature is realized. In principle, a perfect analogical formula with 4D-Einstein equation is applicable to the higher-dimensional general relativity with a modification of gravitational constant (see, for example [16]). Therefore, the gravitational equation in $\{3 T, 3 X\}$-vacuum (in absence of matter) of Geometry (5) reads:

$$
R_{i}^{m}-\frac{1}{2} \delta_{i}^{m} R=-\delta_{i}^{m} \Lambda
$$

where $\Lambda$ is Einstein global cosmological constant; $R_{i}^{m}$ and $R$ are Ricci tensors and the scalar curvature, respectively. Hereafter, for the bi-cylindrical geometry the signatures $\{---+++\}$ are used following a tradition of general relativity in 4D space-time.

Calculating related Christoffel symbols, in according to (6), as $\psi=\psi(y)$ and $\varphi=\varphi(y)$ are not independent variables, we assume that the Hubble law of the cosmological expansion is applicable for the bi-cylindrical model of microscopic space-time: $\frac{\partial \psi}{\partial y}=v_{y}=H_{y} \psi$, where $v_{y}$ is expansion 
rate being proportional to the "microscopic scale factor" $\psi$, while $H_{y}$ is the "microscopic Hubble constant". The symbolical bi-variable $y \equiv\left\{y_{i}, y_{j}\right\}$ is applied for a shorten description of real curved time-space variables. In a time-space separated form the notations of coordinates $\left\{y_{i}\right\}$ or $\left\{y_{j}\right\}$ are time-like or space-like, corresponding to the notations for the symmetrical curved time-space: $\{3 T, 3 X\} \equiv\left\{\psi\left(t_{0}, t_{k}\right), \varphi\left(t_{0}, t_{k}\right), t_{k} \mid \psi\left(x_{n}, x_{l}\right), \varphi\left(x_{n}, x_{l}\right), x_{l}\right\}$. Indeed, $y$ are bi-variables, because they present time-space pairs of similar functions of corresponding time-like and space-like variables in our model. Therefore: $y \equiv\{t, z\} \equiv\left\{t_{0}, t_{3}, x_{n}, x_{3}\right\} \in\left\{t_{i}, x_{j}\right\}$; while $y_{3} \equiv\left\{t_{3}, x_{3}\right\} \in\left\{t_{k}, x_{l}\right\}$ as being implicitly embedded in 3D-time and in 3D-space, respectively. Consequently, as $\psi(y)$ is an exponential solution with a linear combination of its power $\varphi(y)$ in $(6)$, then $\psi(y)$ is separable in relation to different independent variables $y$. In particular, for a fixed amplitude of the cylindrical radius $\psi_{0}$ the time-space separable solution would be: $\psi(y)=\psi_{i}\left(y_{i}\right) \psi_{j}\left(y_{j}\right)=\psi_{0} e^{\varphi\left(y_{i}\right)+\varphi\left(y_{j}\right)}=$ $\psi_{0}\left(e^{H_{y_{i}} y_{i}}+e^{H_{y_{j}} y_{j}}\right)$. Therefore, for partial differentiation, it is expressed:

$$
\left[\frac{\partial y}{\partial \psi}\right]=\frac{1}{H_{y} \psi} \text {. }
$$

The following Christoffel symbols are found valid:

$$
\begin{gathered}
\Gamma_{\varphi \varphi}^{\psi}=-\frac{g^{\psi \psi}}{2} \frac{\partial g_{\varphi \varphi}}{\partial \psi}=-\frac{1}{H_{y}} \frac{\partial \psi}{\partial y} \equiv-\left(\frac{1}{H_{y_{i}}} \frac{\partial \psi}{\partial y_{i}}+\frac{1}{H_{y_{j}}} \frac{\partial \psi}{\partial y_{j}}\right) ; \\
\Gamma_{\psi \varphi}^{\varphi}=\Gamma_{\varphi \psi}^{\varphi}=\frac{g^{\varphi \varphi}}{2} \frac{\partial g_{\varphi \varphi}}{\partial \psi}=\frac{1}{\psi^{2} H_{y}} \frac{\partial \psi}{\partial y} \equiv \frac{1}{\psi^{2}}\left(\frac{1}{H_{y_{i}}} \frac{\partial \psi}{\partial y_{i}}+\frac{1}{H_{y_{j}}} \frac{\partial \psi}{\partial y_{j}}\right) ; \\
\Gamma_{\varphi \varphi}^{3}=-\frac{g^{33}}{2} \frac{\partial g_{\varphi \varphi}}{\partial y_{3}}=-\psi \frac{\partial \psi}{\partial y_{3}} \equiv-\psi\left(\frac{\partial \psi}{\partial t_{3}}+\frac{\partial \psi}{\partial x_{3}}\right) \equiv-\psi\left(\frac{\partial \psi}{\partial t_{k}}+\frac{\partial \psi}{\partial x_{l}}\right) ; \\
\Gamma_{3 \varphi}^{\varphi}=\Gamma_{\varphi 3}^{\varphi}=\frac{g^{\varphi \varphi}}{2} \frac{\partial g_{\varphi \varphi}}{\partial y_{3}}=\frac{1}{\psi} \frac{\partial \psi}{\partial y_{3}} \equiv \frac{1}{\psi}\left(\frac{\partial \psi}{\partial t_{3}}+\frac{\partial \psi}{\partial x_{3}}\right) \equiv \frac{1}{\psi}\left(\frac{\partial \psi}{\partial t_{k}}+\frac{\partial \psi}{\partial x_{l}}\right) ;
\end{gathered}
$$

The tensor curvatures are calculated for the $3 \mathrm{D}$-local cylindrical geometry as

$$
R_{3}^{3}(y)=g^{33} R_{33}=-\frac{1}{\psi} \frac{\partial^{2} \psi}{\partial y_{3}^{2}}=R_{3}^{3}\left(t_{3}\right)+R_{3}^{3}\left(x_{3}\right)
$$

where

$$
\begin{gathered}
R_{3}^{3}\left(t_{3}\right)=\frac{1}{\psi} \frac{\partial^{2} \psi}{\partial t_{3}^{2}} \equiv \frac{1}{\psi} \frac{\partial^{2} \psi}{\partial t_{k}^{2}} \quad \text { and } \quad R_{3}^{3}\left(x_{3}\right)=-\frac{1}{\psi} \frac{\partial^{2} \psi}{\partial x_{3}^{2}} \equiv-\frac{1}{\psi} \frac{\partial^{2} \psi}{\partial x_{l}^{2}} \\
R_{\psi}^{\psi}(y)=g^{\psi \psi} R_{\psi \psi}=-\frac{1}{\psi^{3} H_{y}^{2}} \frac{\partial^{2} \psi}{\partial y^{2}}+\frac{1}{\psi^{4} H_{y}^{2}}\left(\frac{\partial \psi}{\partial y}\right)^{2}=R_{\psi}^{\psi}\left(y_{i}\right)+R_{\psi}^{\psi}\left(y_{j}\right)
\end{gathered}
$$

where

and

$$
R_{\psi}^{\psi}\left(y_{i}\right)=\frac{1}{\psi^{3} H_{y_{i}}^{2}} \frac{\partial^{2} \psi}{\partial y_{i}^{2}}-\frac{1}{\psi^{4} H_{y_{i}}^{2}}\left(\frac{\partial \psi}{\partial y_{i}}\right)^{2}
$$

$$
R_{\psi}^{\psi}\left(y_{j}\right)=-\frac{1}{\psi^{3} H_{y_{j}}^{2}} \frac{\partial^{2} \psi}{\partial y_{j}^{2}}+\frac{1}{\psi^{4} H_{y_{j}}^{2}}\left(\frac{\partial \psi}{\partial y_{j}}\right)^{2}
$$




$$
R_{\varphi}^{\varphi}(y)=g^{\varphi \varphi} R_{\varphi \varphi}=-\frac{1}{\psi^{3} H_{y}^{2}} \frac{\partial^{2} \psi}{\partial y^{2}}+\frac{1}{\psi^{4} H_{y}^{2}}\left(\frac{\partial \psi}{\partial y}\right)^{2}-\frac{1}{\psi} \frac{\partial^{2} \psi}{\partial y_{3}^{2}}=R_{\varphi}^{\varphi}\left(y_{i}\right)+R_{\varphi}^{\varphi}\left(y_{j}\right),
$$

where

and

$$
R_{\varphi}^{\varphi}\left(y_{i}\right)=\frac{1}{\psi^{3} H_{y_{i}}^{2}} \frac{\partial^{2} \psi}{\partial y_{i}^{2}}-\frac{1}{\psi^{4} H_{y_{i}}^{2}}\left(\frac{\partial \psi}{\partial y_{i}}\right)^{2}+\frac{1}{\psi} \frac{\partial^{2} \psi}{\partial t_{3}^{2}}
$$

$$
R_{\varphi}^{\varphi}\left(y_{j}\right)=-\frac{1}{\psi^{3} H_{y_{j}}^{2}} \frac{\partial^{2} \psi}{\partial y_{j}^{2}}+\frac{1}{\psi^{4} H_{y_{j}}^{2}}\left(\frac{\partial \psi}{\partial y_{j}}\right)^{2}-\frac{1}{\psi} \frac{\partial^{2} \psi}{\partial x_{3}^{2}} .
$$

It is to mention that in (18) - (20) all 3D local crossing derivatives within a 3D-subspace are able to make vanished due to the local orthogonality of those coordinates. Even functions $\psi$ and $\varphi$ are extended in both 3D-time and 3D-space, all possible $\{i, j\}$ mixed derivatives between time-like and space-like coordinates are also compensated automatically, because they would be produced in mixing Ricci tensors $R_{\psi\left(y_{i}\right)}^{\psi\left(y_{j}\right)}$ or $R_{\varphi\left(y_{i}\right)}^{\varphi\left(y_{j}\right)}$, however, those mixing tensors are vanished due to diagonality of metrics $g^{i j}$ in the cylindrical geometry. In the result, the symmetrical time-space Ricci tensors $R_{i}^{m}$ in (12) contain only diagonal terms of squared derivatives. Obviously, scalar curvature reads:

$$
R(y)=g^{n i} R_{n i}=g^{n i} g_{n m} R_{i}^{m}=\delta_{m}^{i} R_{i}^{m}=-\frac{2}{\psi^{3} H_{y}^{2}} \frac{\partial^{2} \psi}{\partial y^{2}}+\frac{2}{\psi^{4} H_{y}^{2}}\left(\frac{\partial \psi}{\partial y}\right)^{2}-\frac{2}{\psi} \frac{\partial^{2} \psi}{\partial y_{3}^{2}} .
$$

Its time-space separable form reads: $R(y)=R\left(y_{i}\right)+R\left(y_{j}\right)=\delta_{\alpha}^{\beta} R_{\alpha}^{\beta}\left(y_{i}\right)+\delta_{\gamma}^{\sigma} R_{\gamma}^{\sigma}\left(y_{j}\right)$; where Ricci tensors with $\alpha, \beta \in\{i\}$ are time-like $(\alpha, \beta=1 \div 3)$ and ones with $\gamma, \sigma \in\{j\}$ are space-like $(\gamma, \sigma=$ $1 \div 3$ )

When cosmological constant is vanished $(\Lambda=0)$ the gravitational $\{3 T, 3 X\}$ equation (12) turns into a separable time-space symmetrical representation:

$$
\left[R_{\alpha}^{\beta}\left(y_{i}\right)-\frac{1}{2} \delta_{\alpha}^{\beta} R\left(y_{i}\right)\right]+\left[R_{\gamma}^{\sigma}\left(y_{j}\right)-\frac{1}{2} \delta_{\gamma}^{\sigma} R\left(y_{j}\right)\right]=0 .
$$

Therefore, Eq. (22) is equivalent to a system of component equations:

$$
\begin{aligned}
& R_{3}^{3}(y)=0, \\
& R_{\psi}^{\psi}(y)=0, \\
& R_{\varphi}^{\varphi}(y)=0 .
\end{aligned}
$$

Equation (25) is a trivial relation which is clear from Relations (18) - (20) that: $R_{\varphi}^{\varphi}=R_{\psi}^{\psi}+R_{3}^{3}$, then there only two sub-equations (23) and (24) are independent.

In particular, Eq. (23) in details reads:

$$
R_{3}^{3}(y)=R_{3}^{3}\left(t_{3}\right)+R_{3}^{3}\left(x_{3}\right)=\frac{1}{\psi} \frac{\partial^{2} \psi}{\partial t_{3}^{2}}-\frac{1}{\psi} \frac{\partial^{2} \psi}{\partial x_{3}^{2}} \equiv R_{3}^{3}\left(t_{k}\right)+R_{3}^{3}\left(x_{l}\right)=\frac{1}{\psi} \frac{\partial^{2} \psi}{\partial t_{k}^{2}}-\frac{1}{\psi} \frac{\partial^{2} \psi}{\partial x_{l}^{2}}=0
$$

Then from (26) we obtain a plane wave equation

$$
-\frac{\partial^{2} \psi}{\partial t_{3}^{2}}+\frac{\partial^{2} \psi}{\partial x_{3}^{2}}=0 .
$$


Indeed, Eq. (27) has a wave-like solution $\psi=\psi_{0} e^{\mp i\left(\omega_{3} t_{3}-k_{3} x_{3}\right)}$ which leads to an additional Lorentzlike condition:

$$
\left(\omega_{3}^{2}-k_{3}^{2}\right) \psi=0 .
$$

which compensates any longitudinal fluctuations. Therefore, Eq. (27) defines conservation of linear translation (CLT) which originates from Eq. (3).

The second independent equation (24) determines a bi-geodesic equation

$$
R_{\psi}^{\psi}(y)=R_{\psi}^{\psi}\left(y_{i}\right)+R_{\psi}^{\psi}\left(y_{j}\right)=\frac{1}{\psi^{3} H_{y_{i}}^{2}} \frac{\partial^{2} \psi}{\partial y_{i}^{2}}-\frac{1}{\psi^{4} H_{y_{i}}^{2}}\left(\frac{\partial \psi}{\partial y_{i}}\right)^{2}-\frac{1}{\psi^{3} H_{y_{j}}^{2}} \frac{\partial^{2} \psi}{\partial y_{j}^{2}}+\frac{1}{\psi^{4} H_{y_{j}}^{2}}\left(\frac{\partial \psi}{\partial y_{j}}\right)^{2}=0 .
$$

It leads to an original symmetrical equation

$$
\frac{\partial^{2} \psi}{\partial y_{i}^{2}}-\left(\frac{\partial \psi}{\partial y_{i}}\right)^{2}=\frac{\partial^{2} \psi}{\partial y_{j}^{2}}-\left(\frac{\partial \psi}{\partial y_{j}}\right)^{2} .
$$

For real $\{y\} \equiv\left\{y_{i}, y_{j}\right\}$, Eq. (30) having an exponential solution $\psi \sim e^{H_{y} \cdot y}=e^{\varphi}=e^{\Omega t-k_{j} x_{j}}$ is getting

$$
\frac{\partial^{2} \psi}{\partial t^{2}}-\left[\left(\frac{\partial \varphi}{\partial t_{0}}\right)^{2}+\left(\frac{\partial \varphi}{\partial t_{3}}\right)^{2}\right] \psi=\frac{\partial^{2} \psi}{\partial x_{j}^{2}}-\left[\left(\frac{\partial \varphi}{\partial x_{n}}\right)^{2}+\left(\frac{\partial \varphi}{\partial x_{l}}\right)^{2}\right] \psi .
$$

As differentials $d t_{3}$ and $d t_{0}$, as well as corresponding covariant derivatives are locally orthogonal to each other, their second derivatives are combined together as

$$
\frac{\partial^{2} \psi}{\partial t^{2}}=\frac{\partial^{2} \psi}{\partial t_{0}^{2}}+\frac{\partial^{2} \psi}{\partial t_{3}^{2}}
$$

Similarly, due to a local orthogonality, for differentials $d x_{l}$ and $d x_{n}$, the second derivatives in 3D-space are also combined:

$$
\frac{\partial^{2} \psi}{\partial x_{j}^{2}}=\frac{\partial^{2} \psi}{\partial x_{n}{ }^{2}}+\frac{\partial^{2} \psi}{\partial x_{l}^{2}} .
$$

For a homogeneity condition, Eq. (31) is getting a symmetrical equation of bi-geodesic acceleration of deviation $\psi$ in 3D-time and 3D-space as obtained in [14]:

$$
\frac{\partial^{2} \psi}{\partial t_{0}^{2}}-\left(\frac{\partial \varphi}{\partial t_{0}}\right)^{2} \psi=\frac{\partial^{2} \psi}{\partial x_{n}^{2}}-\left(\frac{\partial \varphi}{\partial x_{n}}\right)^{2} \psi
$$

Recalling that due to 3D local geodesic condition when $\Lambda=0$, both sides in (32) are getting independent and lead to de Sitter-like exponential sub-solutions which describe Hubble-like expansion in microscopic local 3D-time or local 3D-space, correspondingly:

$$
\begin{gathered}
\frac{\partial^{2} \psi}{\partial t_{0}^{2}}-\left(\frac{\partial \varphi}{\partial t_{0}}\right)^{2} \psi \Rightarrow \frac{\partial^{2} \psi}{\partial t_{0}^{+2}}-\left(\frac{\partial \varphi}{\partial t_{0}^{+}}\right)^{2} \psi=\frac{\partial^{2} \psi}{\partial t_{0}^{+^{2}}}-\Lambda_{T} \psi=0, \\
\frac{\partial^{2} \psi}{\partial x_{n}^{2}}-\left(\frac{\partial \varphi}{\partial x_{n}}\right)^{2} \psi \Rightarrow \frac{\partial^{2} \psi}{\partial x_{n}{ }^{2}}-\left(\frac{\partial \varphi}{\partial x_{n}}\right)^{2} \psi=\frac{\partial^{2} \psi}{\partial x_{n}^{2}}-\Lambda_{X} \psi=0,
\end{gathered}
$$


where effective strong potentials $V_{T}$ of a time-like "cosmological constant" $\Lambda_{T}$ with a minor space-like component $\Lambda_{X}$ fulfill breaking symmetry, making time oriented toward the future $\left(t_{0}^{+}-\right.$ signature) and space getting longitudinal polarization ( $x_{n}$-signature of a fixed P-even spin projection). In [15] we suggested that Eq. (33) can fit a scenario similar to the standard cosmological model to formulate a so-called microscopic cosmological model which is able to predict comoving volumes, then calculating masses of charged leptons. Therefore, the mass hierarchy problem of leptons would be solved, based on time-space symmetry.

Equation (31) with the CLT principle and Lorentz-like condition (28) leads to the symmetrical exponential geodesic solution in according to Geometry (5) as follows

$$
-\frac{\partial^{2} \psi}{\partial t^{2}}+\frac{\partial^{2} \psi}{\partial x_{j}^{2}}=-\left[\left(\frac{\partial \varphi}{\partial t_{0}}\right)^{2}-\left(\frac{\partial \varphi}{\partial x_{n}}\right)^{2}\right] \psi
$$

In principle, variables $\{y\}$ can turn as well as $\{y\} \leftrightarrow\{i y\}$ in a mathematical transformation, then Condition (13) turns to:

$$
\left[\frac{\partial y}{\partial \psi}\right]=\frac{-i}{H_{y} \psi}
$$

In the result, Eq. (35) leads to another representation of a wave-like solution with $\psi(y \rightarrow i y) \sim$ $e^{i \varphi}=e^{i\left(\Omega t-k_{j} x_{j}\right)}$ as follows

$$
-\frac{\partial^{2} \psi}{\partial t^{2}}+\frac{\partial^{2} \psi}{\partial x_{j}^{2}}=\left[\left(\frac{\partial \varphi}{\partial t_{0}}\right)^{2}-\left(\frac{\partial \varphi}{\partial x_{n}}\right)^{2}\right] \psi
$$

If time-space symmetry is absolute, the right side is vanished and Eq. (37) turns to:

$$
-\frac{\partial^{2} \psi}{\partial t^{2}}+\frac{\partial^{2} \psi}{\partial x_{j}^{2}}=0
$$

Being involved in metrics $g_{\varphi \varphi}$ the functional parameter $\psi$ characterizes time-space curvatures. Then Eq. (38) would describe a specific kind of microscopic gravitational waves transmitting with the speed of light. However, the time-space symmetry can never be absolute: we have already assumed that the acceleration term in 3D-time is dominantly enhanced due to interaction with a Higgs-like potential, that will produce a time-space asymmetrical polarization $P \rightarrow P^{+}$.

Qualitatively, the original $\{3 T, 3 X\}$ time-space symmetry is broken spontaneously:

$$
\left(V_{T} P\right)^{2}=\left[V_{T}\left(\frac{\partial \varphi}{\partial t_{0}^{-}}+\frac{\partial \varphi}{\partial t_{0}^{+}}\right)\right]^{2} \psi \equiv\left[f_{e}\left(\chi+\phi_{0}\right)\right]^{2} \psi \Rightarrow\left(P^{+}\right)^{2}=\left(\frac{\partial \varphi}{\partial t_{0}^{+}}\right)^{2} \psi \equiv\left(f_{e} \phi_{0}\right)^{2} \psi=m_{0}^{2} \psi,
$$

where $\chi$ is Higgs field and $\phi_{0}$ is Higgs vacuum; $f_{e}$ is Higgs-lepton coupling constant. The arrow means the moment of fixing polarization, equivalent to a spontaneous breaking of symmetry. Since that the elementary particle as a material point has been involved in an almost absolute time-like cylindrical evolution along the real time $t$. Any human observation along the same local geodesic in 3D-time cannot distinguish any spiral evolution because the internal curvature of a cylinder is zero. This is the reason explaining why the physical time axis of a freely moving elementary particle is linear in 4D-Minkowski space-time. 
After spontaneous breaking of time-space symmetry, Eq. (35) determines an asymmetrical bigeodesic equation with exponential solutions in according to Geometry (7):

$$
\frac{\partial^{2} \psi}{\partial t^{2}}-\frac{\partial^{2} \psi}{\partial x_{j}^{2}}=\left[\Lambda_{T}-\left(\frac{\partial \varphi}{\partial x_{n}}\right)_{\text {even }}^{2}-\Lambda_{L}\right] \psi
$$

where $\Lambda_{L} \equiv\left(\frac{\partial \varphi}{\partial x_{n}^{L}}\right)^{2}$ is a small space-like P-odd "cosmological constant" caused by the global weak interaction leading to the left-handed space. Being originated from Einstein gravitational equation (12), Eq. (40) describes the microscopic cosmological evolution of time-space curvatures by its de Sitter-like exponential solutions $\psi=\psi_{0} e^{ \pm \varphi}=\psi_{0} e^{ \pm\left(\Omega t+k_{j} x_{j}\right)}$.

Correspondingly, the wave-like equation (37) with $\psi_{w}(y) \equiv \psi(i y)=\psi_{0} e^{ \pm i \varphi}=\psi_{0} e^{\mp i\left(\Omega t-k_{j} x_{j}\right)}$ due to breaking symmetry leads to:

$$
-\frac{\partial^{2} \psi}{\partial t^{2}}+\frac{\partial^{2} \psi}{\partial x_{j}^{2}}=\left[\left(\frac{\partial \varphi}{\partial t_{0}^{+}}\right)^{2}-B_{e}\left(k_{n} \cdot \mu_{e}\right)_{\text {even }}^{2}-\left(\frac{\partial \varphi}{\partial x_{n}^{L}}\right)^{2}\right] \psi .
$$

where $B_{e}$ is a calibration factor and $\mu_{e}$ is magnetic dipole moment of charged lepton; its orientation is in correlation with spin vector $\vec{s}$ and being P-even.

\section{INTERPRETATION OF PHYSICAL REALITY IN QUANTUM MECHANICS}

In mathematical transformation from the exponential solution to the wave-like one, we should change the signature in Eq. (40), keeping the wave equation (41) mathematically equivalent to the former. This is realized by transformation of variables: $t \rightarrow-i t$ and $x_{j} \rightarrow i x_{j}$, as well as of their corresponding covariant derivatives: $\frac{\partial f}{\partial t} \rightarrow i \frac{\partial f}{\partial t}$ and $\frac{\partial f}{\partial x_{j}} \rightarrow-i \frac{\partial f}{\partial x_{j}}$, similarly as being adopted for quantum dynamic operators. This procedure is not only a mathematical formalism, but also a significant physical operation, equivalent to transformation from an external observation to an internal investigation. Really, it is a fact in quantum mechanics that the phase velocity in the internal phase continuum is superluminal as clear from $\varphi=\Omega t-k_{j} x_{j}=$ const, then $v_{\text {phase }}=\frac{d x_{j}}{d t}=\frac{\Omega}{k_{j}}=\frac{E}{p_{j}}>c$. Somehow, it is equivalent to converting the role of space $\Leftrightarrow$ time in the internal superluminal frame comparing with the external subluminal space-time. Indeed, instead of the real time in 4D-Minkowski geometry one can use an imaginary time in the corresponding $4 \mathrm{D}$ pseudo-Euclid representation. The latter with space-time $\{x,-i t\}$ is explicitly symmetrical in a mathematical transformation $\{x,-i t\} \Leftrightarrow\{i x, t\}$, but for an observation in the subluminal frame the imaginary coordinate is equivalent to a time axis, while the "real time" can be accepted as a spatial axis. Therefore, by rescaling dynamic action with Planck constant (namely, implementing quantum dynamical operators $\frac{\partial}{\partial t} \rightarrow \hat{E}=i \hbar \frac{\partial}{\partial t}$ and $\frac{\partial}{\partial x_{j}} \rightarrow \hat{p}_{j}=-i \hbar \frac{\partial}{\partial x_{j}}$ ) and making the amplitude of the functional parameter $\psi$ of a scale of Compton length, Klein-Gordon-Fock equation in quantum mechanics is to be formulated explicitly from the wave-like solution (41) of Einstein gravitational equation (22) in the extended time-space of sub-Geometry (8) as:

$$
-\hbar^{2} \frac{\partial^{2} \psi}{\partial t^{2}}+\hbar^{2} \frac{\partial^{2} \psi}{\partial x_{j}^{2}}-m^{2} \psi=0
$$


where the square mass term $m$ consists of the following components: $m^{2}=[\hbar \Omega]^{2}=m_{0}^{2}-\delta m^{2}=$ $m_{0}^{2}-m_{s}^{2}-m_{L}^{2}$. Applying Fourier transformation from the phasic space to momentum representation, Eq. (42) reads

$$
E^{2} \psi_{p}-\vec{p}^{2} \psi_{p}-m^{2} \psi_{p}=0
$$

It leads to the relation $E^{2}-\vec{p}^{2}=m^{2}>0$, then Eq. (43) describes subluminal motion of an elementary particle with energy $E$ and momentum $\vec{p}$. In comparison with the traditional expression of the rest mass, the present one includes an additional correction $\delta m$ associated with the contribution of the intrinsic spin in 3D-space. The P-even contribution $m_{s}$ linked with an external curvature of spinning in 3D-space can be compensated in according to 3D-spatial local geodesic condition in Equations (40) and (34) when only the linear translation along $x_{l}$ axis is taken in account for a laboratory frame observation. However, due to P-odd effect being observable in the weak interaction, the geodesic deviation of the material point by its spinning still induces a small non-zero mass factor $m_{L} \ll m_{s}$ which proves a tiny internal curvature of our realistic 3D-space. The latter, in similar to the time-like cylindrical curvature of real time $t$, is not observable from the human point of view of 4D space-time observers, being involved as well in the same global internal cylindrical curvature caused by the weak interaction. In general, Eq. (42) is reminiscent of the squared Dirac equation of lepton [13]. In case when there is no polarization analysis, $m \rightarrow m_{0}$, Eq. (42) turns to the traditional Klein-Gordon-Fock equation in the linear 3D-space of sub-Geometry (9) (with $\left.\left\{x_{j}\right\} \rightarrow\left\{x_{l}\right\}\right)$

$$
-\hbar^{2} \frac{\partial^{2} \psi}{\partial t^{2}}+\hbar^{2} \frac{\partial^{2} \psi}{\partial x_{l}^{2}}-m_{0}^{2} \psi=0
$$

The consistency between the physical reality of an individual elementary particle and the quantum statistical interpretation is a dilemma causing an unsolved philosophical problem. Our proposed model of bi-cylindrical geometrical dynamics would contribute to understanding some issues of this matter. First of all, it gives a meaning of the traditional quantum dynamical operators as timespace converting transformation in the phasic continuum, where the phase velocity of a massive particle is always faster than light. The specific kind of gravitational waves carrying the functional variable $\psi$ of metrics $g_{\varphi \varphi}$ along with in the phasic continuum should be superluminal in this context, and it would be a reason why ones cannot observe directly the quantum wave function $\psi$ except its squared amplitude.

Secondly, it would shed light on the wave-particle duality of quantum mechanics. Qualitatively, the wave-like sub-equation (41) leads to description of a quantum substance as a non-local object with quantum wave features in 4D space-time. On other side, the exponential solution (40) dually describes the same object, but as a material point, i.e. a localized particle following a classical geodesics in an extended time-space.

Thirdly, from the homogeneous $\{3 T, 3 X\}$ bi-geodesic condition (32) it is possible to derive Heisenberg inequalities from space-time curvatures as shown in [14]. Indeed, there was proved that the quantum indeterminism is found to originate from time-like and space-like curvatures, namely, the time-energy inequality is caused by an intrinsic curvature of 3D-time, while the space-momentum inequality is caused by a P-even contribution of spinning in 3D-space of an individual elementary particle. 


\section{CONCLUSION}

Based on time-space symmetry a geometrical dynamical model was developed with an intrinsic cylindrical curvature determined by the functional parameter $\psi$. According to this geometry, Einstein gravitational equation in vacuum has a duality: an exponential solution and another wave-like representation. As it is able to derive Klein-Gordon-Fock equation of a massive elementary particle from the wave-like solution, it was found that quantum mechanics is a special technique with its quantum dynamical operators for describing the superluminal microscopic gravitational waves in the microscopic phase continuum carrying energy-momentum in the corresponding subluminal macroscopic space-time. On other side, the exponential solution leads to a geodesic description of an elementary particle, in particular a massive lepton, as a material point in the extended time-space. In combination, the dual solutions could shed light on origin of the quantum indeterminism [14] and the wave-particle duality. The present geometrical dynamical interpretation of quantum mechanics is in a qualitative agreement with more general conclusions of the STM group [10,11]. For a more quantitative approach, as in a homogeneity condition the geodesic equation is equivalent to de Sitter-like solutions then it serves for modeling Hubble expansion in the microscopic time-space, in analogue to the standard model of macroscopic cosmology. In particular, the proposed microscopic cosmological model with an extension of time-like EDs to the 3D time-like configurations should correlate strictly with the number three of lepton generations which has been used to solve the mass hierarchy problem of charged leptons [15]. Consequently, findings from time-space symmetry of microscopic substances would demonstrate a deep consistency between quantum mechanics and general relativity.

\section{ACKNOWLEDGMENT}

The author thanks Nguyen Anh Ky (Institute of Physics, VAST) and Do Quoc Tuan (Physics Faculty, Hanoi University of Natural Sciences) for useful discussions. A heart-felt thank is also extended to N. B. Nguyen (Thang Long University) for technical assistance.

\section{REFERENCES}

[1] T. Kaluza, Sitz. Preuss. Akad. Wiss. 33 (1921) 966.

[2] O. Klein, Z. f. Physik 37 (1926) 895.

[3] L. de Broglie, J.Phys. et Radium 8 (1927) 225.

[4] D. Bohm, Phys. Rev. 85 (1952) 166.

[5] J. S. Bell, Physics 1 (1964) 195.

[6] S. J. Freedman and J. F. Clauser, Phys. Rev. Lett. 28 (1972) 938.

[7] J. Maldacena, Adv.Theor.Math.Phys. 2 (1998) 231.

[8] L. Randall and R. Sundrum, Phys. Rev. Lett. 83 (1999) 4690.

[9] P. S. Wesson, Phys. Lett. B 276 (1992) 299.

[10] S. S. Seahra, P. S. Wesson, Gen. Rel. Grav. 33 (2001) 1731.

[11] P. S. Wesson, Phys. Lett. B 722 (2013) 1.

[12] B. Koch, arXiv:0801.4635v2[quant-ph]2009.

[13] Vo Van Thuan, IJMPA 24 (2009) 3545.

[14] Vo Van Thuan, Com. Phys. 25 (2015) 247, arXiv:1507.00251[gr-qc]2015.

[15] Vo Van Thuan, arXiv:1510.04126[physics.gen-ph]2015.

[16] P. S. Wesson, Space-Time-Matter: Modern higher-dimensional cosmology. World Scientific, 2nd Edition, Singapore, 2007. 